64 IS AN END OF LIFE CARE SURVEY AT THE BEREAVEMENT APPOINTMENT ACCEPTABLE TO CARERS?

Hannah McLoughlin, Kay Stewart, James Davies, Andrea Hanson. Sheffield Teaching Hospitals

\subsection{6/bmjspcare-2018-ASPabstracts.91}

Aim To explore the feasibility and acceptability of bereavement survey.

Methods We surveyed deceased patients' next of kin (NOK) on the care they felt their relative received in their last admission to hospital. The survey was conducted from July 2016 to June 2017 within a large teaching hospital. A 28 question survey focused on communication with the patient and those important to them, management of their symptoms, care and dignity shown to the patient, fluids and nutrition at end of life, and care of those persons important to the patient. This mirrored the local and national end of life care guidance. The survey was initially posted to the deceased patients registered NOK 2-6 weeks after death. However it became apparent that the details for the NOK were not fully completed in the hospital records and this only allowed contact with 39\% of NOK. From September 2016 it was decided to trial distributing the survey at the bereavement appointment, this is the time when the NOK receives the death certificate. The survey was paired with a covering letter detailing bereavement support information and self-addressed envelope for return. Maternal and neonatal deaths, suicide, cases accepted by the coroner and deaths in $\mathrm{A}$ and $\mathrm{E}$ were excluded. The team also used their discretion when handing out surveys and if they felt it would complicate a person's grief they excluded them.

Results 297 completed surveys were returned over the 12 month period. There was only one complaint regarding the survey itself. This complaint related to a survey sent at 6 weeks.

There were no verbal complaints to any staff members involved in the survey process and the process was acceptable to staff.

Conclusion This study suggests that it is acceptable to carers and staff to sensitively distribute a bereavement survey at the bereavement appointment.

\section{$65 \quad$ QUALITY OF DEATH OF RENAL PATIENTS DYING IN ACUTE HOSPITAL - DOES RENAL SUPPORTIVE CARE (RSC) LEAD TO BETTER DEATHS?}

Kat Urban, Celine Foote, Frank Brennan, Mark Brown, Jenny Burman, Brendon Lee. Concord Repatriation General Hospital, St. George Hospital

\subsection{6/bmjspcare-2018-ASPabstracts.92}

Background Patients with chronic kidney disease, whether on dialysis or not, frequently die in the acute hospital and have a high symptom burden. In New South Wales, a state-wide RSC service has been established to provide palliative support to nephrology services.

Null hypothesis No significant improvement in indicators of quality of death at 5 and 10 years after establishment of RSC services.

Methods Retrospective chart review of all deaths in the acute hospital under a Nephrology service in the years 2004 (establishment of RSC), 2009 and 2014 at St George Hospital (SGH) and in 2009 (establishment of RSC) and 2014 at
Concord Repatriation General Hospital (CRGH). There is a plan to assess deaths in 2019 at this site. Domains assessed were recognition of dying, avoidance of invasive interventions, symptom assessment, anticipatory prescribing, documentation of spiritual needs and bereavement information for families. End of Life Care Plan use was also evaluated at STG.

Results At SGH over 90\% of patients were recognised to be dying in all 3 years. CPR rates did not differ between the 3 years. There was an increase in the documentation of respiratory secretions $(p=0.01)$. Rates of interventions in the last week of life did not differ. Use of EOLCPs significantly increased over the 3 years $(p=0.01)$. There was a significant increase in prescription of anti-psychotic $(\mathrm{p}=0.008)$, antiemetic $(p=0.002)$ and anti-cholinergic $(p=0.03)$ medication.

At $\mathrm{CRGH}$ there was no significant change in any domain from 2009 to 2014. There was a non significant trend towards improved recognition of dying (63\% to 92\%).

Conclusion There was an increase in the use of EOLCPs and anticipatory prescribing at SGH, but not at $\mathrm{CRGH}$, which may mean that 10 years are required after the establishment of RSC services before real changes to patient care are seen.

\section{AUDITING THE COMPLETION OF DOCUMENTATION FOR PATIENTS APPROACHING THE END OF LIFE}

Thomas Prew, Andrew Davies, Katherine Webber, Jo Thompson. Royal Surrey County Hospital

10.1136/bmjspcare-2018-ASPabstracts.93

Background Royal Surrey County Hospital (RSCH) has implemented a Personalised End of Life Care Plan (PELiCan) designed to assess the needs and symptom control of patients in the last seven days of life. This audit aimed to assess how regularly and thoroughly the documents were completed.

Method PELiCan documents were reviewed for all patients who died in $\mathrm{RSCH}$ with a PELiCan in place from 1 August to 30 September 2017. Of particular interest were: completion of the Personal Care Plan on Day One; next of kin contact details documentation; and Medical Day One Assessment, which reviewed whether patient and carers were aware of the terminal prognosis and whether preferred place of death (PPD) had been discussed. Multidisciplinary team entries were also audited to confirm that the Supportive and Palliative Care Team (SPCT) had reviewed patients daily, including on weekends and Bank Holidays. Also, drug charts were assessed for key medications in symptom management.

Results 68 patients died with PELiCan pathways in place. 63 of $68(92.65 \%)$ were reviewed daily by the SPCT between starting a PELiCan and their death. Contact details were recorded in only 44 of 68 documents $(64.71 \%)$, and PPD, in 46 of 68 documents (67.65\%). However, the carer was documented as aware of the prognosis in 63 of 68 documents (92.65\%).

Conclusion The trust met its internal target (90\%) for SPCT reviews and carer notification of prognosis, but fell short in documenting PPD. Reviewing the notes, it is feasible that important discussions with families were taking place, but remained insufficiently documented. The authors intend this audit to be developed into a quality improvement project which will familiarise ward teams with PELiCan documentation and the most important areas for completion. The audit will then be repeated. 